\title{
Elemental analysis
}

Elementalanalysis (\%) calcd for poly(AAMPSO$\left.{ }_{3} \mathrm{H}\right) \mathrm{MBAA}$ : C 38.59, H 5.87, N 7.61, S16.59; Found: C 40.76, H 5.1, N 6.6, S 16.21.

Elementalanalysis (\%) calcd for poly(AE-SO3) MBAA: C 28.05, H 3.30, N 0.36, S 14.40; Found: C 30.03, H 2.91, N 1.39, S 15,42.

Elementalanalysis (\%) calcd for poly(AE-TMA) MBAA: C 50.70, H 8.46, N 7.59; Found: C 45.76, H 8.1, N 6.62.

Elementalanalysis (\%) calcd for poly(HPMAA) MBAA: C 56.89, H 8.71, N 11.20; Found: C 55.41, H 8.11, N 8.66.

Elementalanalysis (\%) calcd for poly(MAEDMA-SO $)$ MBAA: C 46.38, H 7.39, N 5.49, S 12.08; Found: C 50.21, H 7.11, N 6.62, S 13.49.

Elementalanalysis (\%) calcd for poly(MAE-SO 3 ) MBAA: C 32.11, H 4.04, N 0.36, S 13.80; Found: C 31.41, H $6.11, \mathrm{~N} 2.62, \mathrm{~S} 12.02$

Elementalanalysis (\%) calcd for poly(MAE-TMA) MBAA: C 53.14, H 8.87, N 7.10; Found: C 50.89, H 8.90, N 6.18.

Elementalanalysis (\%) calcd for poly(VEtImBr) MBAA: C 42.49, H 5.58, N 13.15; Found: C 41.83, H 7.34, N 12.91.

Elementalanalysis (\%) calcd for poly(TMA-VB) MBAA: C 69.16, H 8.70, N 6.98; Found: C 71.41, H 8.11, N 7.62.

Elementalanalysis (\%) calcd for poly(MPC) MBAA: C 45.84, H 7.64, N 5.10; Found: C 47.26, H 6.74, N 4.93.

Elementalanalysis (\%) calcd for poly(HEMA) MBAA: C 56.46, H 7,88, N 0.36; Found: C 55.67, H 6.29, N 1.42.

Elementalanalysis (\%) calcd for poly(MAE-TMA) 700 PEGDA: C 52.11, H 8.73, N 6.61; Found: C 51.73, H 8.90, N 7.72 . 


\section{NMR of the synthesized monomers}

VEtImBr: ${ }^{1} \mathrm{H}$ NMR $\left[250 \mathrm{MHz}\right.$, acetone- $d_{6}$ with a drop of methanol- $d_{3}, \delta / \mathrm{ppm}$ relative to tetramethylsilane (TMS)]:10.20 (s, 1H), 8.25 (s, 1H), $8.02(\mathrm{~s}, 1 \mathrm{H}), 7.53(\mathrm{dd}, 1 \mathrm{H}), 6.17$ (dd, 1H), 5.47 (dd, 1H), 4.53 (q, $2 \mathrm{H}), 1.64$ (t, 3H). ${ }^{13} \mathrm{C}$ NMR $[250 \mathrm{MHz}$, acetone-d6,ס]: 206.68, 128.77, 122.98, 119.48, 108.65, 45.23, 14.44 .

HPMAA: ${ }^{1} \mathrm{H}$ NMR $\left[250 \mathrm{MHz}, \mathrm{CDCl}_{3}, \delta / p p m\right.$ relative to tetramethylsilane (TMS)]:1.16 (d, 3H), $1.92(\mathrm{~m}, 3 \mathrm{H}), 3.12(\mathrm{~m}$, 1H), $3.38(\mathrm{br}, 1 \mathrm{H}), 3.45(\mathrm{~m}, 1 \mathrm{H}), 3.90(\mathrm{~m}, 1 \mathrm{H}), 5.30(\mathrm{~m}, 1 \mathrm{H}), 5.68(\mathrm{~m}, 1 \mathrm{H}), 6.49(\mathrm{br}, 1 \mathrm{H})$. 